\title{
HIGHER DIMENSIONAL ENRIQUES VARIETIES WITH EVEN INDEX
}

\author{
JIN HONG KIM
}

(Communicated by Lev Borisov)

\begin{abstract}
Let $Y$ be an Enriques variety of complex dimension $2 n-2$ with $n \geq 2$. Assume that $n=2 m$ for odd prime $m$. In this paper we show that $Y$ is the quotient of a product of a Calabi-Yau manifold of dimension $2 m$ and an irreducible holomorphic symplectic manifold of dimension $2 m-2$ by an automorphism of order $n$ acting freely. We also show that both $Y$ and its universal cover are always projective.
\end{abstract}

\section{INTRODUCTION AND MAIN RESULTS}

A compact complex smooth Kähler manifold $X$ is called irreducible symplectic if $X$ is simply connected and $H^{0}\left(X, \Omega_{X}^{2}\right)$ is generated by a nowhere vanishing holomorphic 2-form. It can be considered as a higher dimensional analogue of $K 3$ surfaces (see [5] for more details). Every automorphism of finite order on $K 3$ surfaces without fixed points is a non-symplectic involution, and their quotients are known to be the Enriques surfaces (see [1]).

Recently, in their paper [4] Boissière, Nieper-Wisskirchen, and Sarti introduced the notion of an Enriques variety which is a higher dimensional analogue of the Enriques surface. To be precise, a compact complex smooth Kähler manifold $Y$ of dimension $2 n-2$ with $n \geq 2$ is called an Enriques variety if its canonical divisor $K_{Y}$ has order $n$ in the Picard group $\operatorname{Pic}(Y)$, the holomorphic Euler characteristic $\chi\left(Y, \mathcal{O}_{Y}\right)$ is equal to 1 , and the fundamental group $\pi_{1}(Y)$ of $Y$ is cyclic of order $n$. Even more generally, $Y$ is called a weak Enriques variety if for some divisor $d$ of $n$, $K_{Y}$ has order $d$ in $\operatorname{Pic}(Y), \chi\left(Y, \mathcal{O}_{Y}\right)=n / d$, and $\pi_{1}(Y)$ is cyclic of order $d$. As in the papers [8] and [4], the order of the fundamental group $\pi_{1}(Y)$ of $Y$ will be called the index of $Y$.

The study of automorphisms of $K 3$ surfaces was essentially initiated by the work [6] of Nikulin, and since then much progress has been made. One of the primary motivations to consider the Enriques variety as in our paper is, in fact, to study the automorphisms of irreducible holomorphic symplectic manifolds (see [2], 3], and [7] for some earlier works).

Received by the editors September 20, 2011 and, in revised form, January 6, 2012 and January $11,2012$.

2010 Mathematics Subject Classification. Primary 14E05, 14J28, 14J32, 14J40.

Key words and phrases. Enriques varieties, Calabi-Yau manifolds, holomorphic symplectic manifolds, index. 
The aim of this paper is to give some more refined results on Enriques varieties with even index which have been motivated by Proposition 2.1 in the paper 4 . of Boissière, Nieper-Wisskirchen, and Sarti. To be more precise, in the paper [4] Boissière, Nieper-Wisskirchen, and Sarti essentially proved the following theorem (Proposition 2.1 of [4]).

Theorem 1.1. Let $Y$ be an Enriques variety of complex dimension $2 n-2$ with $n \geq 2$. If $n$ is equal to 2 or an odd integer, then $Y$ is the quotient of an irreducible holomorphic symplectic manifold by a fixed point free automorphism of order $n$. In particular, $Y$ is projective.

In the same paper [4, Boissière-Nieper-Wisskirchen-Sarti also gave a counterexample to Theorem 1.1 in the case when $n \geq 4$ is even. Their counterexample is a 10dimensional Enriques variety which is the quotient of a product of a 6-dimensional Calabi-Yau manifold and a 4-dimensional irreducible holomorphic symplectic manifold by an automorphism of order 6 which splits and acts freely (see Section 4.3 of 4 for more details). Roughly speaking, results of our paper show that essentially there can be no other types of counterexamples in higher dimensions. More precisely, our main result is

Theorem 1.2. Let $Y$ be an Enriques variety of complex dimension $2 n-2$ with $n \geq 2$. Assume that $n=2 m$ for prime $m$. Then the following assertions hold:

(a) If $m$ is equal to 2 , then $Y$ is either the quotient of an irreducible symplectic holomorphic manifold of complex dimension 6 by an automorphism $f$ of order 4 acting freely or the quotient of a product of a Calabi-Yau manifold of complex dimension 4 and a Calabi-Yau manifold of complex dimension 2 (or a K3 surface) by an automorphism $f$ of order 4 acting freely.

(b) If $m$ is odd prime, then $Y$ is the quotient of a product of a Calabi-Yau manifold of complex dimension $2 m$ and an irreducible holomorphic symplectic manifold of complex dimension $2 m-2$ by an automorphism $f$ of order $n$ acting freely.

Remark 1.3. (a) In their paper [8], Oguiso and Schröer defined an Enriques manifold to be a compact complex manifold that is not simply connected and whose universal cover is an irreducible holomorphic symplectic manifold. So an Enriques variety of complex dimension $2 n-2$ whose fundamental group $\pi_{1}(Y)$ is cyclic of order $n=2 m$ with odd prime $m$ is not an Enriques manifold in the sense of Oguiso and Schröer.

(b) We do not know if the automorphism $f$ in Theorem 1.2 always splits so that $Y$ decomposes into a product which already contains an Enriques variety.

It is generally believed that all Enriques varieties should always be projective. In view of this belief, we also show the following theorem in Section 3 .

Theorem 1.4. Let $Y$ be an Enriques variety of complex dimension $2 n-2$ with $n \geq 2$. Assume that $n=2 m$ for prime $m$. Then both $Y$ and its universal cover are always projective.

We organize this paper as follows. In Section 2, we shall give a proof of Theorem 1.2 Section 3 is devoted to proving Theorem 1.4 


\section{Proof of Theorem 1.2}

The goal of this section is to provide a proof of Theorem 1.2 whose argument is based on the Bogomolov decomposition theorem of compact Kähler manifolds with the first Chern class $c_{1}=0$.

Proof of Theorem 1.2. Assume first that $m$ is odd prime. Since $K_{Y}$ has order $n$ in $\operatorname{Pic}(Y)$, there exists a finite unramified covering $\pi: X \rightarrow Y$ of order $n$ so that $K_{X}=$ $\pi^{*} K_{Y}$ is trivial. By assumption, the fundamental group $\pi_{1}(Y)$ is cyclic of order $n$. So $X$ is actually the universal covering of $Y$. Since $K_{X}=\pi^{*} K_{Y}$ is trivial, the first Chern class $c_{1}(X)$ is also zero. Hence, it follows from the Bogomolov decomposition theorem of compact Kähler manifolds with $c_{1}=0$ that $X$ is isomorphic to a product

$$
T \times \prod_{i} V_{i} \times \prod_{j} W_{j}
$$

where $T$ is a complex torus, $V_{i}$ is a Calabi-Yau manifold, and $W_{j}$ is an irreducible holomorphic symplectic manifold.

Now note that

$$
n=\chi\left(X, \mathcal{O}_{X}\right)=\chi\left(T, \mathcal{O}_{T}\right) \prod_{i} \chi\left(V_{i}, \mathcal{O}_{V_{i}}\right) \times \prod_{j} \chi\left(W_{j}, \mathcal{O}_{W_{j}}\right),
$$

where we used $\chi\left(X, \mathcal{O}_{X}\right)=n \times \chi\left(Y, \mathcal{O}_{Y}\right)=n$ in the first equality. But, if $\operatorname{dim}_{\mathbb{C}} V_{i}$ is odd, then $\chi\left(V_{i}, \mathcal{O}_{V_{i}}\right)=0$. Hence $\operatorname{dim}_{\mathbb{C}} V_{i}$ is even. Moreover, if $\operatorname{dim}_{\mathbb{C}} T$ is greater than 1 , then $\chi\left(T, \mathcal{O}_{T}\right)=0$. On the other hand, if $\operatorname{dim}_{\mathbb{C}} T$ is equal to 1 , then the complex dimension of $X$ not only becomes odd but is also not simply connected. Therefore, there are no complex torus and no Calabi-Yau manifolds with odd complex dimension in the decomposition (2.1). Recall also the well-known fact that

$$
\chi\left(W_{j}, \mathcal{O}_{W_{j}}\right)=\frac{\operatorname{dim}_{\mathbb{C}} W_{j}}{2}+1 \geq 2 .
$$

With this understood, we now obtain

$$
n=2 m=\chi\left(X, \mathcal{O}_{X}\right)=2^{t}\left(\frac{w_{1}}{2}+1\right) \cdots\left(\frac{w_{s}}{2}+1\right),
$$

where $t$ denotes the number of Calabi-Yau manifolds of even complex dimension in the decomposition (2.1) and $w_{j}$ denotes the complex dimension of $W_{j}$. Since $m$ is assumed to be an odd prime, it follows from (2.2) that $0 \leq t \leq 1$. If $t$ is equal to 1 , then we have

$$
\begin{aligned}
m & =\left(\frac{w_{1}}{2}+1\right) \cdots\left(\frac{w_{s}}{2}+1\right), \\
2 n-2 & =4 m-2=v_{1}+w_{1}+\cdots+w_{s},
\end{aligned}
$$

where $v_{i}$ denotes the complex dimension of $V_{i}$. Since $m$ is an odd prime, $s$ is equal to 1 by the first equation of (2.3), and $v_{1}=2 m$ and $w_{1}=2 m-2$. On the other hand, if $t$ is equal to 0 , then there exists an $i$, say 1 , such that $\frac{w_{i}}{2}+1=2$ and $s=2$. Moreover, it is easy to obtain that $4 m-4=w_{2}=2 m-2$, which implies $m=1$. This is a contradiction. This completes the proof of Theorem 1.2 (b).

Next, we deal with the case when $m=2$. To do so, we first consider the case when $t$ is equal to 2. Then it follows from (2.2) that we have $s=0$. By taking into account the dimension of $X$, we also have $v_{1}+v_{2}=6$. Since $t$ is the number of Calabi-Yau manifolds of even complex dimension, there are only two possibilities for $v_{1}$ and $v_{2}$ : either $v_{1}=2$ and $v_{2}=4$ or $v_{1}=4$ and $v_{2}=2$. In either case, $Y$ is the 
quotient of a product of a Calabi-Yau manifold of complex dimension 2 (or a $K 3$ surface) and a Calabi-Yau manifold of complex dimension 4 by an automorphism $f$ of order 4 acting freely. On the other hand, if $t$ is equal to 1 , we have $s=1$ and $w_{1}=2$ by (2.2), and $v_{1}=4$ by the second equation of (2.3). So $Y$ is the quotient of a product of a Calabi-Yau manifold of complex dimension 4 and a Calabi-Yau manifold of complex dimension 2 (or a $K 3$ surface) by an automorphism $f$ of order 4 acting freely.

Finally, if $t$ is equal to 0 , then it follows from (2.2) that we have two possibilities for $s$ : either $s=1$ or $s=2$. If $s$ is equal to 1 , then we have $w_{1}=6$ by (2.2). In this case, $Y$ is simply the quotient of an irreducible symplectic holomorphic manifold of complex dimension 6 by an automorphism $f$ of order 4 acting freely. If $s$ is equal to 2 , then we have $w_{1}=w_{2}=2$ by (2.2), and thus $w_{1}+w_{2}=4$. But this is not equal to the complex dimension of $X$ that is equal to 6 . So this case does not occur. This completes the proof of Theorem 1.2 (a).

This completes the proof of Theorem 1.2 .

\section{Proof of Theorem 1.4}

In this section, we give a a proof of Theorem 1.4.

Proof of Theorem 1.4. Let $h^{p, q}(Y)=\operatorname{dim}_{\mathbb{C}} H^{q}\left(Y, \Omega_{Y}^{p}\right)$ and let $G$ be the cyclic group generated by the automorphism $f$ in Theorem 1.2. Then it is clear that $H^{0}\left(Y, \Omega_{Y}^{p}\right)=H^{0}\left(X, \Omega_{X}^{p}\right)^{G}$. The proof is divided into three steps:

Step 1. In this step, we first deal with the case where $Y$ is the quotient of a product of a Calabi-Yau manifold $V$ of complex dimension $2 m$ and an irreducible holomorphic symplectic manifold $W$ of complex dimension $2 m-2$ by an automorphism $f$ of order $n$ acting freely. We then show that $H^{0}\left(Y, \Omega_{Y}^{2}\right)=0$. This will be a key ingredient in Step 3 to prove that $Y$ and its universal cover $X$ are projective.

To prove that $H^{0}\left(Y, \Omega_{Y}^{2}\right)=0$, note first from the Künneth formula that we have

$$
H^{0}\left(X, \Omega_{X}^{p}\right) \cong \bigoplus_{r+s=p} H^{0}\left(V, \Omega_{V}^{r}\right) \otimes H^{0}\left(W, \Omega_{W}^{s}\right)
$$

Recall then that for a Calabi-Yau manifold $V, h^{r, 0}(V)=0$ for $0<r<\operatorname{dim}_{\mathbb{C}} V$, while for an irreducible holomorphic manifold $W, h^{s, 0}(W)=0$ for odd $s$ with $0<s<\operatorname{dim}_{\mathbb{C}} W$. Thus, it is easy to obtain that for all $p$ with $\operatorname{dim}_{\mathbb{C}} W<\operatorname{dim}_{\mathbb{C}} V=$ $\operatorname{dim}_{\mathbb{C}} W+2 \leq p \leq \operatorname{dim}_{\mathbb{C}} V+\operatorname{dim}_{\mathbb{C}} W=\operatorname{dim}_{\mathbb{C}} X$

$$
\begin{aligned}
& H^{0}\left(X, \Omega_{X}^{p}\right)=H^{0}\left(V, \Omega_{V}^{\operatorname{dim}_{\mathbb{C}} V}\right) \otimes H^{0}\left(W, \Omega_{W}^{p-\operatorname{dim}_{\mathbb{C}} V}\right) \\
& \cong \begin{cases}\mathbb{C}, & \text { for even } p \text { with } \operatorname{dim}_{\mathbb{C}} V \leq p \leq \operatorname{dim}_{\mathbb{C}} V+\operatorname{dim}_{\mathbb{C}} W, \\
0, & \text { otherwise }\end{cases}
\end{aligned}
$$

On the other hand, for $0 \leq p \leq \operatorname{dim}_{\mathbb{C}} W$ we have

$$
\begin{aligned}
& H^{0}\left(X, \Omega_{X}^{p}\right)=H^{0}\left(W, \Omega_{W}^{p}\right) \\
& \cong \begin{cases}\mathbb{C}, & \text { for even } p \text { with } 0 \leq p \leq \operatorname{dim}_{\mathbb{C}} W, \\
0, & \text { otherwise. }\end{cases}
\end{aligned}
$$

Next we claim that $h^{2,0}(Y)=h^{0,2}(Y)=0$. Indeed, it follows from (3.2) and $\operatorname{dim}_{\mathbb{C}} W \geq 2$ that $H^{0}\left(X, \Omega_{X}^{2}\right)=H^{0}\left(W, \Omega_{W}^{2}\right)$. So let $\sigma$ be a generator of $H^{0}\left(W, \Omega_{W}^{2}\right)$. Then the following lemma holds. 
Lemma 3.1. The automorphism $f$ of $X$ is actually non-symplectic in the sense that there exists a primitive $n$-th root of unity $\xi$ such that the action of $f$ on $1 \otimes \sigma$ in $H^{0}\left(X, \Omega_{X}^{2}\right)$ is given by $f^{*}(1 \otimes \sigma)=\xi(1 \otimes \sigma)$.

Proof. To prove it, suppose that, on the contrary, $f$ is not non-symplectic. Then there would exist some integer $i(1 \leq i \leq n-1)$ such that $f^{i}$ is symplectic, i.e., $\left(f^{i}\right)^{*}(1 \otimes \sigma)=1 \otimes \sigma$. Without loss of generality, we may assume that $i$ is equal to 1.

Since $H^{0}\left(W, \Omega_{W}^{p}\right)$ is zero for odd $p$ and generated by $\sigma^{p / 2}$ for even $p$, it is easy to obtain

$$
\sum_{p=0}^{\operatorname{dim}_{\mathbb{C}} W}(-1)^{p} \operatorname{tr}\left(\left.f^{*}\right|_{H^{0}\left(X, \Omega_{X}^{p}\right)}\right)=\frac{\operatorname{dim}_{\mathbb{C}} W}{2}+1 .
$$

Now, let $\Theta_{V}$ denote a generator of $H^{0}\left(V, \Omega_{V}^{\operatorname{dim}_{\mathbb{C}} V}\right)$. Then we see from (3.1) that there exists some $x \in \mathbb{C}$ such that

$$
f^{*}\left(\Theta_{V} \otimes 1\right)=x \Theta_{V} \otimes 1 .
$$

Since $\Theta_{V} \otimes \sigma^{k}$ is a generator of $H^{0}\left(V, \Omega_{V}^{\operatorname{dim}_{\mathbb{C}} V}\right) \otimes H^{0}\left(W, \Omega_{W}^{k-\operatorname{dim}_{\mathbb{C}} V}\right) \cong \mathbb{C}$ for each $0 \leq k \leq \frac{\operatorname{dim}_{\mathbb{C}} W}{2}$, it follows from (3.4) and the identity $f^{*}(1 \otimes \sigma)=1 \otimes \sigma$ that

$$
f^{*}\left(\Theta_{V} \otimes \sigma^{k}\right)=x \Theta_{V} \otimes \sigma^{k}, \quad 0 \leq k \leq \frac{\operatorname{dim}_{\mathbb{C}} W}{2} .
$$

Hence it is straightforward to obtain

$$
\begin{aligned}
& \sum_{p=\operatorname{dim}_{\mathbb{C}} V}^{\operatorname{dim}_{\mathbb{C}} V+\operatorname{dim}_{\mathbb{C}} W}(-1)^{p} \operatorname{tr}\left(\left.f^{*}\right|_{H^{0}\left(V, \Omega_{V}^{\operatorname{dim}_{\mathbb{C}} V}\right) \otimes H^{0}\left(W, \Omega_{W}^{p-\operatorname{dim}_{\mathbb{C}} V}\right)}\right) \\
= & x\left(\frac{\operatorname{dim}_{\mathbb{C}} W}{2}+1\right) .
\end{aligned}
$$

Now, recall that $\operatorname{dim}_{\mathbb{C}} V=\operatorname{dim}_{\mathbb{C}} W+2$. Thus, if we combine two equations, (3.3) and (3.5), it is immediate to obtain the holomorphic Lefschetz number $L(f)$ of $f$ that is equal to

$$
L(f)=\sum_{p=0}^{\operatorname{dim}_{\mathbb{C}} X}(-1)^{p} \operatorname{tr}\left(\left.f^{*}\right|_{H^{0}\left(X, \Omega_{X}^{p}\right)}\right)=(1+x)\left(\frac{\operatorname{dim}_{\mathbb{C}} W}{2}+1\right) .
$$

At this point, it is important to notice that the only case of symplectic $f$ which would give a vanishing holomorphic Lefschetz number is when $x$ is equal to -1 . More precisely, if $x$ is not equal to -1 , then the holomorphic Lefschetz number $L(f)$ of $f$ is not zero. So $f$ would have a fixed point. But, this contradicts the fact that $f$ acts freely.

Therefore, we assume that $x$ is equal to -1 . Then, by using the same argument as above it is easy to see that the holomorphic Lefschetz number $L\left(f^{2}\right)$ of $f^{2}$ would be equal to

$$
2\left(\frac{\operatorname{dim}_{\mathbb{C}} W}{2}+1\right)=\operatorname{dim}_{\mathbb{C}} W+2
$$

which is clearly non-zero. So $f^{2}$ would have a fixed point. However, again this contradicts the fact that $f$ acts freely. This completes the proof of Lemma 3.1 
Consequently, by Lemma 3.1 we have $f^{*}(1 \otimes \sigma)=\xi(1 \otimes \sigma)$ for some $n$-th root of unity $\xi$. But then clearly $1 \otimes \sigma$ is not invariant under $f$. Hence we have $H^{0}\left(Y, \Omega_{Y}^{2}\right)=$ 0 . This completes the proof of Step 1.

Step 2. In this step, we next consider the case where $Y$ is the quotient of an irreducible symplectic holomorphic manifold $X$ of complex dimension 6 by an automorphism $f$ of order 4 acting freely. In the same way as in Step 1, we can show that $H^{0}\left(Y, \Omega_{Y}^{2}\right)=0$. Indeed, note first that we have

$$
H^{0}\left(X, \Omega_{X}^{p}\right)= \begin{cases}\mathbb{C}, & \text { for even } p \text { with } 0 \leq p \leq \operatorname{dim}_{\mathbb{C}} X=6, \\ 0, & \text { otherwise. }\end{cases}
$$

Let $\sigma$ be a generator of $H^{0}\left(X, \Omega_{X}^{2}\right)$. Then it can be shown exactly as in Step 1 that there exists a primitive 4-root of unity $\xi$ such that $f^{*}(\sigma)=\xi \sigma$; i.e., $f$ is nonsymplectic in the usual sense (refer to Section 2.2 of 4 for more details). This implies that $\sigma$ is not invariant under $f$. Therefore we have $H^{0}\left(Y, \Omega_{Y}^{2}\right)=0$. This completes the proof of Step 2.

Step 3. Finally we prove that both $Y$ and its universal cover $X$ are projective. To do so, since $h^{2,0}(Y)=h^{0,2}(Y)=0$ by Step 1 and Step 2, note first that the inclusion of $H^{1,1}(Y)_{\mathbb{R}}$ into $H^{2}(Y, \mathbb{R})$ is bijective. The fact that the Kähler cone inside $H^{1,1}(Y)_{\mathbb{R}}$ is non-empty and open then implies that there exists an integral class on $Y$. Hence it follows from the Kodaira embedding theorem that the Kähler manifold $Y$ is projective. By pulling back the integral class on $Y$, we can also obtain an integral Kähler class on $X$, proving that $X$ is also projective.

This completes the proof of Theorem 1.4 .

\section{ACKNOWLEDGEMENTS}

The author is grateful to the referee for many valuable comments on an earlier version of this paper. This research was supported by the Basic Science Research Program through the National Research Foundation of Korea (NRF) funded by the Ministry of Education, Science and Technology (2010-0020928, 2011-0025685, 2011-0001181).

\section{REFERENCES}

[1] W. Barth, C. Peters, and A. Van de Ven, Compact complex surfaces, Ergeb. Math. Grenzgebiete 4, Springer, Berlin, 1984. MR749574 (86c:32026)

[2] S. Boissière, Automorphismes naturels de l'espace de Douady de points sur une surface, Canad. J. Math. 64 (2012), 3-23. MR2932167

[3] S. Boissière and A. Sarti, A note on automorphisms and birational transformations of holomorphic symplectic manifolds, Proceedings of Amer. Math. Soc. 140 (2012), no. 12, 4053-4062. MR2957195

[4] S. Boissière, M. Nieper-Wisskirchen, and A. Sarti, Higher dimensional Enriques varieties and automorphisms of generalized Kummer varieties, Journal de Mathématiques Pures et Appliquées 95 (5) (2011), 553-563. MR2786223

[5] M. Gross, D. Huybrechts, and D. Joyce, Calabi-Yau manifolds and related geometries, Springer, Berlin, 2003. MR1963559 (2004c:14075)

[6] V. Nikulin, Finite automorphism groups of Kähler K3 surfaces, Trans. Mosc. Math. Soc. 2 (1980), 71-135. MR0544937 (81e:32033) 
[7] K. Oguiso, Tits alternative in hyperkähler manifolds, Mah. Res. Lett. 13 (2006), 307-316. MR.2231119 (2007e:14023)

[8] K. Oguiso and S. Schröer, Enriques manifolds, J. Reine Angew. Math. 661 (2011), 215-235. $\operatorname{MR} 2863907$

Department of Mathematical Sciences, Korea Advanced Institute of Science and Technology, Yuseong-Gu, Daejeon 305-701, Republic of Korea

Current address: Department of Mathematics Education, Chosun University, 309 Pilmundaero, Dong-gu, Gwangju 501-759, Republic of Korea

E-mail address: jinhkim11@gmail.com 